\title{
Ferramenta para avaliação e gestão da visita domiciliar na atenção primária à saúde: um relato de experiência
}

\section{Tool for home visit evaluation and management in primary health care: an experience report \\ Herramienta para la evaluación y gestión de la visita domiciliaria en atención primaria a la salud: un relato de experiencia}

Juliana Viana Pinheiro1, Marco Túlio Aguiar Mourão Ribeiro², Tatiana Monteiro Fiuza², Renan Magalhaes Montenegro Junior²

${ }^{1}$ Secretaria de Saúde de Fortaleza. Fortaleza, CE, Brasil.

2 Universidade Federal do Ceará. Fortaleza, CE, Brasil.

\section{Resumo}

Introdução: A visita domiciliar permite ao profissional de saúde romper com o modelo centrado na doença, voltando-se para uma abordagem centrada no indivíduo. No entanto, observa-se uma grande deficiência na organização e gestão destas, o que muitas vezes dificulta a priorização mais adequada destes pacientes. Objetivo: Apresentar a experiência de uma equipe ao utilizar uma nova ferramenta para avaliação e classificação de risco que visa otimizar a gestão da agenda das visitas domiciliares. Métodos: Trata-se de um relato de experiência de uma equipe de saúde da família, na periferia de uma capital no Nordeste do Brasil, que aplicou esta ferramenta aos pacientes de visita domiciliar entre os meses de junho e novembro de 2017. Resultados: Os dados coletados foram analisados, sendo possível classificar os pacientes quanto ao risco e vulnerabilidade. Esta ferramenta favoreceu o fortalecimento e estruturação do registro, planejamento, avaliação e monitoramento das visitas realizadas nos território. Além disso, deve ser ressaltada sua fácil aplicação e a reprodutibilidade em diferentes cenários da atenção primária. Conclusão: $O$ uso de instrumentos adequados permite identificar pacientes em situação de risco e vulnerabilidade, além de possibilitar a organização da agenda, a identificação das necessidades das pessoas, o acompanhamento das doenças crônicas, propor estratégias, e planejar intervenções futuras.

Palavras-chave: Serviços de Assistência Domiciliar; Atenção Primária à Saúde; Avaliação em Saúde; Organização e Administração
Como citar: Pinheiro JV, Ribeiro MTAM, Fiuza TM, Montenegro Junior RM. Ferramenta para avaliação e gestão da visita domiciliar na atenção primária à saúde: um relato de experiência. Rev Bras Med Fam Comunidade. 2019;14(41):1818. https://doi.org/10.5712/rbmfc14(41)1818
Autor correspondente: Juliana Viana Pinheiro. E-mail: juliana_vpinheiro@hotmail.com Fonte de financiamento: declaram não haver.

Parecer CEP: não se aplica.

Procedência e revisão por pares: revisado por pares. Recebido em: 17/07/2018. Aprovado em: 28/03/2019. 


\begin{abstract}
Introduction: The home visit allows the health professional to break the disease-centered model, turning to an individual-centered approach. However, there is a great deficiency in the organization and management of these, which often makes it more difficult to prioritize these patients. Objective: To present the experience of a team when using a new risk evaluation and classification tool that aims to optimize the management of the home visits schedule. Methods: This is an report of the experience of a family health team, in a peripheric neighborhood of a capital city in the northeast of Brazil, that applied this tool with patients who applied this tool to home visiting patients in the period between June and November 2017. Results: The data collected were analyzed and it was possible to classify the patients regarding risk and vulnerability. This tool favored the strengthening and structuring of the registration, planning, evaluation and monitoring of the visits made in the territory. In addition, its easy application and reproducibility in different primary care settings should be emphasized. Conclusion: The use of appropriate instruments allows the identification of patients at risk and vulnerability, besides organizing the agenda, identifying people's needs, monitoring chronic diseases, proposing strategies, and planning for future interventions.
\end{abstract}

Keywords: Home Care Services; Primary Health Care; Health Evaluation; Organization and Administration

\title{
Resumen
}

Introducción: La visita domiciliaria permite al profesional de la salud romper con el modelo centrado en la enfermedad, volviéndose hacia un enfoque centrado en el individuo. Sin embargo, se observa una gran deficiencia en la organización y gestión de éstas, lo que a menudo dificulta la priorización más adecuada de estos pacientes. Objetivo: Presentar la experiencia de un equipo en la utilización de una nueva herramienta de evaluación y clasificación de riesgo que visa optimizar la gestión de la agenda de visitas domiciliarias. Métodos: Tratase de un relato de experiencia de un equipo de salud de la familia en la periferia de una capital en el Nordeste de Brasil, donde se aplicó esta herramienta con pacientes asistidos en domicilio entre los meses de junio y noviembre de 2017. Resultados: Los dados obtenidos fueron analizados, siendo posible clasificar según el riesgo y la vulnerabilidad. Esta herramienta favoreció el fortalecimiento y estructuración de registro, planificación, evaluación y monitoreo de las visitas realizadas en el territorio. Además, llamamos la atención a su fácil aplicabilidad y reproductibilidad en diferentes escenarios de la atención primaria a la salud. Conclusión: El uso de instrumentos adecuados permite identificar pacientes en situación de riesgo y vulnerabilidad, además de posibilitar la organización de la agenda, la identificación de las necesidades de las personas, el seguimiento de las enfermedades crónicas, proponer estrategias, y planificar intervenciones futuras.

Palabras clave: Servicios de Atención de Salud a Domicilio; Atención Primaria de Salud; Evaluación en Salud; Organización y Administración

\section{Introdução}

O fenômeno de transição epidemiológica, representado pelo aumento proporcional da carga de doenças crônico-degenerativas e pelo aumento da expectativa de vida, é uma realidade em vários países. O principal fator responsável é a queda das taxas de fecundidade e de mortalidade. ${ }^{1}$

Esta mudança no perfil demográfico gera como consequência uma preocupação com o aumento dos custos com a saúde. Doenças de curso agudo tendem a ser resolvidas de forma mais rápida, gerando demandas mais breves aos serviços de saúde. De outro modo, as doenças crônico-degenerativas se relacionam a prevalências mais altas e longos períodos de cuidado, o que gera demandas mais frequentes e internamentos mais prolongados. ${ }^{2}$

O Brasil, assim como diversos países europeus, está reorganizando de forma gradual seus serviços de saúde, de forma a atender mais adequadamente às necessidades desse novo perfil epidemiológico. ${ }^{2} \mathrm{~A}$ atenção domiciliar vem ganhando espaço em todo o mundo como uma estratégia no cuidado longitudinal desses pacientes.

Além da comprovada efetividade e da consequente redução nos custos com cuidado à saúde, que já foram bem documentados no contexto da atenção primária, a visita domiciliar permite ao profissional assistente romper com o modelo centrado na doença, voltando-se para uma abordagem centrada no indivíduo. Durante o ato assistencial, é possível observar a dinâmica familiar, conhecer o ambiente no qual o paciente está inserido e compreender como tais fatores interferem na saúde do paciente. ${ }^{3}$ 
Apesar da grande importância nos processos de trabalho da atenção primária, a maioria dos profissionais não avaliam e nem estratificam o binômio risco-vulnerabilidade do paciente a ser visitado, assim como não planejam a periodicidade das visitas e quais serão os profissionais responsáveis pelas visitas na estrutura da equipe. Assim, muitas vezes não é possível, apesar dos esforços, respeitar determinados princípios, quais sejam: universalidade, equidade e longitudinalidade, no contexto da assistência domiciliar.

Apesar do marcante desenvolvimento nas últimas duas décadas de cursos de pós-graduação, atualização e educação multiprofissional continuada, ainda há poucos protocolos estabelecidos e divulgados que visem à organização do processo de trabalho das visitas domiciliares.

Diante deste contexto, os autores elaboraram uma ferramenta, tendo como base critérios que serão discutidos no decorrer do artigo, a fim de ajudar a organizar a agenda, identificando as necessidades das pessoas, propondo estratégias e planejando intervenções futuras.

Neste artigo, daremos ênfase à experiência de uma equipe de saúde da familia ao aplicar a escala de risco e vulnerabilidade para avaliação dos seus pacientes para gestão e organização das visitas domiciliares. Esta escala apresenta uma nova proposta para avaliação e gestão da visita domiciliar para atenção primária à saúde, uma vez que estratifica o paciente considerando a condição clínica, fatores de risco e vulnerabilidade específicos, além de avaliar as condições ambientais e avaliação do cuidador. Esta ferramenta possibilita qualificar a assistência, visto que auxilia na avaliação e estratificação de risco e vulnerabilidade dos pacientes a serem visitados, permitindo, dessa forma, o planejamento das visitas futuras no contexto da atenção domiciliar.

\section{Métodos}

Trata-se de um relato de experiência realizado pelos médicos de família (preceptores e residente) que aplicaram esta ferramenta de avaliação de risco e vulnerabilidade aos pacientes acamados ou domiciliados vinculados à equipe Verde no período de junho a novembro de 2017.

A equipe Verde está vinculada à unidade de atenção primária à saúde (UAPS) Lineu Jucá, que é uma unidade docente assistencial, sendo campo de prática para os alunos dos primeiro, segundo e sétimo semestres e internato de Medicina de Família e Comunidade do curso de medicina da Universidade Federal do Ceará, além da Residência de Medicina de Família e Comunidade. Portanto, são realizadas atividades assistenciais, produção científica e ensino. Esta UAPS localiza-se na Barra do Ceará, bairro da periferia de Fortaleza/Ceará.

Inicialmente, foram realizados a territorialização e o diagnóstico de situação da equipe com o auxílio dos agentes de saúde. A população deste estudo faz parte da área de abrangência da equipe, que é responsável por cerca de 4200 pessoas. Foi solicitado aos agentes de saúde que identificassem quantos e quais eram os pacientes acamados ou com dificuldade de locomoção, que teriam necessidade de atendimento domiciliar. A partir da análise de situação, os agentes de saúde detectaram 58 pacientes com este perfil.

Antes de iniciar as visitas, foi feito um planejamentocom a equipe. Desta forma, os seis agentes comunitários, técnica de enfermagem, enfermeiro, médico residente e médico da equipe se reuniram para avaliar o número de pacientes e quais os perfis destes. Além disto, foi necessário definir quantos 
e quais turnos seriam necessários para realizar as visitas, quais profissionais iriam realizar, para que fosse estabelecido um cronograma destas visitas. Para ajudar este planejamento e alinhamento entre os integrantes da equipe, foram feitos os seguintes questionamentos:

Quando visitar?

Qual a frequência das visitas domiciliares na agenda de uma equipe?

Como visitar?

Qual integrante da equipe deve realizar as visitas?

Por que visitar?

Quem visitar?

\section{Quando visitar?}

O período ou turno de visita na agenda deverão ser definidos em equipe, de acordo com a realidade de cada localidade e com a demanda de pacientes que necessitam de VD. A territorialização e análise de situação são o ponto de partida para responder a estas perguntas. Algumas situações devem ser consideradas para se definir turno ou horário para visitas programadas, dentre elas: situação de violência na comunidade e horários mais convenientes, rotina das famílias, disponibilidade de transporte, formas de acesso e pressão assistencial. Destaca-se que, se houver necessidades de visitas para uma demanda aguda ou urgência, o profissional pode e deve realizar em qualquer horário da sua agenda.

\section{Qual a frequência das visitas domiciliares na agenda de uma equipe?}

A análise de situação e o planejamento com a equipe são fundamentais para se organizar a frequência das visitas na equipe. Como o território é dinâmico, a agenda deve se adaptar a eventuais mudanças que podem aparecer, uma vez que a prioridade é facilitar o acesso e garantir um cuidado continuado.

\section{Como visitar?}

Dependendo da distância do território, as visitas podem ser feitas a pé ou de carro. Geralmente, o município disponibiliza um transporte para auxiliar na realização das visitas. A presença do ACS é importante, pois é, na maioria das vezes, o componente da equipe com maior vínculo com o território e com a família, o que ajuda a estreitar o contato do profissional com a família e facilita a entrada da equipe no território. É importante ressaltar que este planejamento leva em consideração o desejo do paciente e de sua família, procura-se compartilhar a responsabilidade do cuidado, proporcionando aumento da autonomia do indivíduo e de seus cuidadores.

\section{Qual integrante da equipe deve realizar as visitas?}

Com a elaboração de um plano de cuidado para cada paciente, incluindo lista de problemas, metas terapêuticas, serviços e equipamentos que precisam ser utilizados, podemos estabelecer qual componente da equipe deve ficar responsável por cada ação proposta. 


\section{Por que visitar?}

Garantir acesso aos serviços de saúde, promovendo ações coordenadas com uma visão integral do indivíduo. Além do já exposto, como conhecer a realidade paciente- meio, garantir acesso aos cuidados de saúde a aqueles que não conseguem receber os cuidados na Unidade Básica de Saúde atende ao princípio da equidade e universalidade do cuidado. Em Fortaleza, cidade em que é elaborado o artigo, alguns pacientes necessitam ser atendidos em domicílio por conflito entre facções. Cabe ao médico de família identificar tais situações para garantir o cuidado de saúde de todos.

\section{Quem visitar?}

Os pacientes serão definidos em reunião de equipe na qual serão classificados de acordo com perfil operacional de atendimento de modalidade da AD. De modo geral, são pacientes com condições clínicas estáveis ou não com algum tipo dificuldade de acesso à unidade de saúde.

Após esta etapa, foi estabelecido, em reunião, um cronograma de visitas domiciliares semanais, no período de junho a novembro de 2017. Para priorizar quais pacientes seriam visitados, inicialmente utilizamos a escala de Savassi e Coelho.

$\mathrm{Na}$ literatura, estão disponíveis alguns questionários para planejamento das visitas domiciliares como a avaliação do Risco e Vulnerabilidade familiar $^{4}$ e Escala de Risco, ${ }^{5}$ que utilizam variáveis como saneamento básico, critérios epidemiológicos e sociais.

Desta forma, foram realizadas as visitas a todos os pacientes, com aplicação prévia da Escala de Savassi e Coelho e a partir da visita foi realizada avaliação clínica e familiar e social deste pacientes.

Apesar destas ferramentas fornecerem um diagnóstico preeliminar dos pacientes que teriam maior vulnerabilidade, a equipe teve dificuldade em realizar esta triagem em territórios homogêneos, em que os determinantes sociais são comuns às famílias. A escala de Savassi e Coelho é extremamente útil para triar ou identificar pacientes vulneráveis ou de risco pelo ACS, no entanto, após a consulta do profissional médico, enfermeiro ou outro profissional de nível superior, percebeu-se a necessidade de incluir outros critérios clínicos, familiares e sociais para melhor avaliação e estratificação do risco e vulnerabilidade destes pacientes.

Como vários dos pacientes em situação de atenção domiciliar são idosos, com várias comorbidades, em uso de várias medicações, em situação de fragilidade e dependencia funcional, os autores elaboraram uma escala conforme descrito no Quadro 1 para avaliação destes pacientes e suas famílias, levando em consideração os seguintes critérios: idade, condição clínica, uso de polifarmácia, funcionalidade, mobilidade, síndrome da fragilidade, suporte familiar e necessidade de cuidados paliativos.

Antes de ser aplicada aos pacientes da equipe, a escala passou por um processo de avaliação e pré-teste. Este instrumento foi apresentado para um grupo de 15 residentes e 12 preceptores do Programa de Residência de Medicina de Família e Comunidade da Universidade Federal do Ceará, sendo feita avaliação por estes atores e ajustes no instrumento. Após isto, foi feita aplicação a 15 pacientes de duas UAPS diferentes, que são campo de estágio do programa de residência, quando foram feitos ajustes dos critérios de risco. Após esta etapa, foram aplicados aos 58 pacientes da equipe. 
Quadro 1. Escala para classificação de risco e vulnerabilidade clínica para pacientes em visita domiciliar na APS.

Nome:

$\mathrm{DN}:$ ______ Idade:

Escala de risco e vulnerabilidade para atenção domiciliar na APS (Ribeiro, Fiuza e Pinheiro)

\begin{tabular}{|c|c|c|c|}
\hline Indicador & Situação & Escore de risco e vulnerabilidade: & Pontuação obtida \\
\hline Idade & $\begin{array}{l}75 \text { a } 84 \text { anos } \\
>85 \text { anos }\end{array}$ & $\begin{array}{l}1 \\
2\end{array}$ & \\
\hline Multimorbidade & $\begin{array}{l}\text { № de comorbidades }(\geq 5) \\
\text { Descompensação clínica }\end{array}$ & $\begin{array}{l}2 \\
5\end{array}$ & \\
\hline Polifarmácia & № de medicamentos $(\geq 5)$ & 2 & \\
\hline Dependência Funcional & $\begin{array}{l}\text { AVDs instrumentais } \\
\text { AVDs Básicas e Instrumentais }\end{array}$ & $\begin{array}{l}1 \\
2\end{array}$ & \\
\hline Mobilidade & $\begin{array}{l}\text { Dificuldade de marcha } \\
\text { Risco de queda } \\
\text { Acamado }\end{array}$ & $\begin{array}{l}1 \\
2 \\
3\end{array}$ & \\
\hline Suporte familiar & $\begin{array}{l}\text { Disfunção familiar } \\
\text { Sobrecarga do cuidador }\end{array}$ & $\begin{array}{l}1 \\
1\end{array}$ & \\
\hline Fragilidade & $\begin{array}{l}\text { Síndrome demencial, } \\
\text { depressão, } \\
\text { Parkinson, neoplasia, } \\
\text { sarcopenia, } \\
\text { desnutrição, } \\
\text { disfagia, } \\
\text { incontinência, } \\
\text { Paralisia cerebral }\end{array}$ & 2 ( cada) & \\
\hline Cuidados Paliativos & $\begin{array}{l}\text { PPS }\left(^{*}\right) 80 \text { A } 100 \\
\text { PPS } 50 \text { A } 70 \\
\text { PPS } 30 \text { A } 50 \\
\text { PPS < } 20 \\
{ }^{*} \text { PPS: Palliative Performance } \\
\text { Scale }\end{array}$ & $\begin{array}{c}2 \\
5 \\
8 \\
10\end{array}$ & \\
\hline Total & & & \\
\hline
\end{tabular}

* PPS: Palliative Performance Scale: Escala de Performance Paliativa (PPS) é uma ferramenta amplamente utilizada para avaliação e classificação de cuidados paliativos, sendo um instrumento validado para o contexto e lingua portuguesa em $2009^{6}$

A Escala de Desempenho em Cuidados Paliativos versão 2 ( EDCP v2) é de autoria da "Victoria Hospice Society" Disponível em formato Word eletrônico sob pedido pelo e-mail edu.hospice@viha.ca. Correspondências devem ser enviadas ao diretor Clínico, Victoria Hospice Society, 1952 Bay Street, Victoria BC, V8R 1J8, Canada.

De acordo com a pontuação obtida na escala, podemos classificar o risco e vulnerabilidade de cada pessoa e planejar as próximas visitas, conforme descrito na Tabela 1:

\section{Resultados e Discussão}

As visitas foram realizadas com a presença do médico preceptor, residente de MFC, alunos do internato em Saúde Comunitária e agente de saúde. Para cada um dos 58 pacientes, identificados em junho de 2017, em situação de visita domiciliar, foi aplicado o instrumento proposto de avaliação e estratificação de risco e vulnerabilidade. 
Tabela 1. Classificação de risco e vulnerabilidade, de acordo com a pontuação e planejamento temporal das próximas visitas.

\begin{tabular}{lcc} 
Classificação de risco e vulnerabilidade & Escore & $\begin{array}{c}\text { Tempo médio para planejamento das } \\
\text { próximas visitas }\end{array}$ \\
\hline Baixo & Até 5 & 6 meses a 1 ano \\
Médio & 6 a 10 & 4 a 6 meses \\
Alto & 11 a 15 & 2 a 3 meses \\
Muito alto & Maior que 16 & 1 a 2 meses \\
\hline
\end{tabular}

Observou-se que os dados desta escala podem ser obtidos com uma anamnese e exame físico bem feitos. Percebeu-se que não foi necessário realizar nenhuma conduta diferente da habital ou solicitar exames para poder aplicar o novo instrumento.

Após a avaliação e estratificação de risco e vulnerabilidade, os autores elaboraram uma proposta de planejamento através desta avaliação e classificação.

É importante ressaltar que a abordagem centrada na pessoa deve ser o norte para este planejamento; de acordo com a comorbidade e com a situação de cada paciente, os profissionais terão autonomia para agendar os retornos. Este planejamento das visitas serve como um parâmetro ou referência para apoiar a gestão das VD pela equipe. A seguir, apresentamos o consolidado das pessoas avaliadas e classificadas, assim como uma proposta de planejamento de cada visita, conforme descrito na Tabela 2.

Tabela 2. Planejamento das visitas através da classificação de risco e vulnerabilidade, de acordo com a pontuação e planejamento temporal das próximas visitas.

\begin{tabular}{lcc} 
Classificação de risco e vulnerabilidade & Número de pessoas & $\begin{array}{c}\text { Tempo médio para planejamento das } \\
\text { próximas visitas }\end{array}$ \\
\hline Baixo & 05 & 6 meses a 1 ano \\
Médio & 21 & 4 a 6 meses \\
Alto & 22 & 2 a 3 meses \\
Muito alto & 10 & 1 a 2 meses \\
\hline
\end{tabular}

A equipe de médicos que aplicou esta ferramenta considerou-a adequada e pertinente ao fortalecimento e estruturação do registro, planejamento, avaliação e monitoramento das visitas realizadas nos território. Além disso, o planejamento permitiu atender a demanda reprimida e reorganizar a agenda. A fácil aplicação e a reprodutibilidade em diferentes cenários da atenção primária também foram ressaltados pela equipe.

Além desta classificação, foi possível identificar os problemas mais prevalentes e relevantes das pessoas em situação de atendimento domiciliar. Conhecer as afecções mais frequentes é uma das competências do médico de família para, dessa forma, possibilitar o cuidado mais adequado aos problemas mais frequentes da comunidade em que atua.

Este instrumento foi apresentado a outros médicos da atenção primária e está sendo aplicado a outras equipes do programa de Residência de Medicina de Família, sendo avaliado como um instrumento de grande relevância para gestão das visitas domiciliares, assim como para ajudar a avaliação clínica e familiar dos pacientes de atenção domiciliar da atenção primária.

Outro ganho observado com a melhor gestão da agenda da equipe é respeitar os princípios norteadores do SUS como a facilidade de acesso, equidade, longitudinalidade e coordenação do cuidado. 


\section{Conclusão}

Com as mudanças na estrutura etária da população e a tendência de ampliação do cuidado na atenção primária, as visitas domiciliares se tornam cada vez mais uma necessidade, e o uso racional do tempo e dos recursos para a sua realização precisam de instrumentos de gestão.

O uso de instrumentos adequados permite identificar pacientes em situação de risco e vulnerabilidade. Além de apoiar os médicos de família e comunidade e outros profissionais de saúde da atenção primária na organização da agenda, identificação das necessidades das pessoas, acompanhamento das doenças crônicas, propor estratégias e planejar intervenções futuras.

A ferramenta aqui proposta serve como apoio, sendo o escore de resultado um guia para a gestão das visitas domiciliares na atenção primária à saúde. Porém, a decisão final é do profissional que aplica a ferramenta ao individualizar as escolhas aos pacientes que ele já conhece, levando em consideração a autonomia do paciente.

O instrumento não foi avaliado por outros seviços, limitando este artigo como um relato de experiência. Sugerimos que este instrumento seja aplicado por outras equipes, em outros municípios e outras regiões do país, para que sejam feitos outros estudos e artigos para validá-lo.

\section{Contribuição dos autores}

Concepção e/ou delineamento do estudo: Ribeiro MTAM, Fiuza TM, Pinheiro JV. Aquisição, análise ou interpretação dos dados: Ribeiro MTAM, Fiuza TM, Pinheiro JV, Montenegro Junior RM. Redação preliminar: Ribeiro MTAM, Pinheiro JV. Revisão crítica da versão preliminar: Ribeiro MTAM, Fiuza TM, Pinheiro JV, Montenegro Junior RM.

Todos os autores aprovaram a versão final e concordaram com prestar contas sobre todos os aspectos do trabalho.

\section{Conflito de interesses}

Declaram não haver.

\section{Referências}

1. Dos Reis CS, Noronha K, Wajnman S. Envelhecimento populacional e gastos com internação do SUS: uma análise realizada para o Brasil entre 2000 e 2010. Rev Bras Estud Popul. 2016;33(3):591-612. https://doi.org/10.20947/S0102-30982016c0007

2. Carneiro LAF, Campino ACC, Leite F, Rodrigues CG, dos Santos GMM, Silva ARA. Envelhecimento populacional e os desafios para o sistema de saúde brasileiro. São Paulo: Instituto de Estudos de Saúde Complementar, IESS; 2013.

3. Albuquerque ABA, Bosi MLM. Visita Domicilar no âmbito da Estratégia Saúde da Família: percepções do usuário no Município de Fortaleza, Ceará, Brasil. Cad Saúde Pública. 2009;25(5):1103-112. https://doi.org/10.1590/S0102-311X2009000500017

4. Fiúza TM, Ribeiro MTAM. Sensibilidade no diagnóstico de desnutrição protéico-calórica realizado pelas equipes de saúde por meio do SIAB e de busca ativa de casos. Rev Bras Med Fam Comunidade. 2007;3(9):21-6. https://doi.org/10.5712/rbmfc3(9)79

5. Savassi LCM, Lage JL, Coelho FLG. Sistematização de instrumento de estratificação de risco familiar: a Escala de Risco Familiar de Coelho-Savassi. J Mang Prim Health Care. 2012;3(2):179-85.

6. Maciel MGS, Carvalho RT. Palliative Performance Scale PPS Versão 2. Tradução brasileira para a língua portuguesa. São Paulo; 2009. 